\title{
It all started with a visit to the emergency department
}

The articles in this summer supplement span the spectrum of medical conditions from psychosis to trauma, but all start from the same place: the emergency room.

In the emergency department (ED), teams of healthcare providers must be prepared for anything. While the cases in this issue were ultimately managed by many departments and specialists, the ED teams were on the front lines of care management and were responsible for appropriately coordinating care with other specialists. These articles remind us that each patient is unique, and that their medical management requires personalized plans, be they surgical, psychiatric, medical, or a combination of treatment modalities.

These case reports are not your typical day at the office. First, a patient repeatedly visits the $\mathrm{ED}$ with nonspecific abdominal symptoms to be eventually diagnosed with a rare case of mesenteric chylous lymphangioma. In the next report, a unique case of psychosis is mitigated by treating suspected underlying attention deficit hyperactivity disorder. Finally, a 29-year-old man suffers severe hypothermic cardiac arrest after a car crash, but is successfully reanimated.

It is important to appreciate our fellow students for documenting these unique cases. They are disseminating the medical knowledge gained through clinical experiences and educating others about the quick decisions that ED teams and specialists made to change patient outcomes for the better. It is also important that we recognize the exceptional educational role undertaken by their supervising clinicians, who helped facilitate the first-hand clinical experiences.

Read on to discover the interesting cases with which students of the Schulich School of Medicine \& Dentistry were involved over the summer of 2015.

\section{- Ramona Neferu}

Junior Associate Editor 